Revista de Psicología y Educación / Journal of Psychology and Education, 2020, 15(1), 30-45 (www.rpye.es) Doi: https://doi.org/10.23923/rpye2020.01.184

ISSN: $1699-9517$

\title{
Ansiedad escénica musical en profesorado de conservatorio: frecuencia y análisis por género

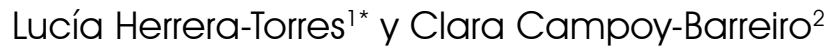 \\ Universidad de Granada \\ ${ }^{2}$ Instituto de Educación Secundaria Rusadir
}

\begin{abstract}
Resumen: La ansiedad escénica musical es un problema al que se suele enfrentar la mayoría de los intérpretes musicales, tanto profesorado como alumnado. La literatura científica indica que su manifestación puede diferir según diferentes variables. Es por ello, que el presente estudio examina, por un lado, si el profesorado de Conservatorio ha experimentado ansiedad escénica musical al enfrentarse a sus actuaciones y, por otro, si la ansiedad escénica musical difiere en función del género. Para ello, se ha utilizado un estudio ex-post-facto de tipo comparativo-causal, en el que ha participado un total de 46 docentes de Conservatorio de Málaga, Sevilla y la ciudad autónoma de Melilla (España). Para la recogida de información se utilizó el Inventario de Ansiedad Escénica Musical de Kenny (K-MPAl), adaptado al español. Además, se incluyeron una serie de preguntas sociodemográficas así como algunas preguntas adicionales relativas a la ansiedad escénica musical. Tras el análisis de los datos, los resultados indican que el $80.4 \%$ de la muestra ha padecido ansiedad escénica musical en algún momento de su carrera musical. Igualmente, las mujeres puntúan más alto que los hombres en diferentes ítems. Finalmente, se discuten diferentes propuestas para la prevención e intervención de la ansiedad escénica musical.
\end{abstract}

Palabras clave: Ansiedad escénica musical, Profesorado, Conservatorio, Género.

\section{Music Performance Anxiety in Conservatory Teachers: Frequency and analysis by gender}

\begin{abstract}
Music performance anxiety is a problem that most musical performers, both teachers and students, face. The scientific literature indicates that its manifestation can differ according to different variables. It is for this reason that the present study examines, on the one hand, if teachers of the Conservatory experienced music performance anxiety in the face of their actions and, on the other, if the music performance anxiety makes differences according to the gender variable. For it, a comparative-causal ex-post-facto study has been developed, in which a total of 46 teachers from the Malaga, Seville and the autonomous city of Melilla's Conservatory in Spain participated. Kenny's Musical Anxiety Inventory (K-MPAl) adapted to Spanish was used to collect information. In addition, a series of sociodemographic questions were included as well as some additional questions related to the music performance anxiety. After the data analysis, the results indicate that $80.4 \%$ of the sample had experienced stage-wide anxiety at some point in their musical career. Likewise, female score higher than male in different items. Finally, different proposals to prevent and to treat the music performance anxiety are discussed.
\end{abstract}

Keywords: Music performance anxiety, Teachers, Conservatory, Gender.

La Psicología de la Música es un enfoque que desde el comienzo del siglo XX se encarga de analizar la carga emocional que ejerce la música en el comportamiento de la persona a lo largo de su vida. Más recientemente, una nueva corriente toma importancia en el estudio de las emociones desde otra

Recibido: 26/09/2018 - Aceptado: 23/06/2019 - Avance online: 27/09/2019 *Correspondencia: Lucía Herrera Torres.

Universidad de Granada.

C.P: 52005, Melilla, España.

E-mail: luciaht@ugr.es

Herrera-Torres, L. y Campoy-Barreiro, C. (Avance Online). Ansiedad escénica musical en profesorado de conservatorio: frecuencia y análisis por género. Revista de Psicología y Educación, 15(1), 32-47, hitps://doi.org/10.23923/rpye2020.01.184 perspectiva: aquéllas que afectan al músico antes, durante y después de enfrentarse a una actuación en público (Hallam, Cross, \& Thaut, 2011 ). La realización de un concierto o recital puede inducir sentimientos asombrosamente positivos en instrumentistas y cantantes. Pueden convertirse en la razón que les estimula para repetir dicha experiencia una y otra vez sin importar las horas de práctica que se hayan invertido. Thomas Allen (barítono inglés) formuló las siguientes palabras a un periodista al finalizar una obra de Don

1699-9517/@ 2020 Asociación Científica de Psicología y Educación (ACIPE). Publicado por Consejo General de Colegios Oficiales de Psicólogos, España. Este es un artículo Open Access bajo la CC BY-NC-ND licencia (http://creativecommons.org/licencias/bync-nd/4.0/1. 
Giovanni en Japón: "Podría haber caminado sobre los techos de las casas, estaba en la cima" (Ballester, 2015, p. 258).

Sin embargo, al igual que fructífera, la carrera musical puede convertirse en una carrera amarga y de sin sabores ya que las actuaciones musicales también pueden generar emociones negativas tales como ansiedad o miedo. Éstas, pueden llegar a resultar devastadoras a nivel interpretativo, mermando las habilidades del intérprete y pudiendo hacer que éste pierda el control de la obra (Salmon, 1990; Zarza, Casanova, \& Orejudo, 2016). Cuando dichas emociones interfieren de forma negativa en la interpretación, puede llegar a convertirse en un trastorno llamado ansiedad escénica (Dalia, 2004).

La ansiedad es un estado de ánimo negativo caracterizado por síntomas corporales de tensión física y aprensión con respecto al futuro (Amerian Psychiatric Association, 1994). Dicho estado de ánimo suele anticipar daño o desgracia futuros y su objetivo puede ser interno o externo. La ansiedad en sí es una señal de alerta que advierte sobre un peligro inminente y que tiene como objetivo ayudar a la persona a que adopte las medidas necesarias para enfrentarse a una amenaza. Suele estar acompañada de una serie de síntomas que se manifiestan a nivel físico, cognitivo, conductual y social. Cierto grado de ansiedad es incluso deseable para el manejo óptimo de las exigencias cotidianas. Ahora bien, cuando ésta sobrepasa cierta intensidad o supera la capacidad adaptativa de la persona, se convierte en patológica, provocando un malestar o deterioro significativo de la actividad del individuo donde pueden aparecer o acentuarse los síntomas anteriormente descritos (Herrera \& Jorge, 2013; Kenny \& Ackermann, 2015; Papageorgi, Creech, \& Welch, 2011 ).

La ansiedad escénica es una patología que sufre una gran parte de la población en general, por ejemplo, al exponerse a situaciones donde tienen que enfrentarse a un público (Zarza, Casanova, \& Robles, 2016). Los síntomas que se padecen suelen ser náuseas, sudoración excesiva y temblores. En su forma más aguda, puede incluso llegar a paralizar a la persona afectada (Papageorgi, Hallam, \& Welch, 2007). En el caso de los músicos, cuya profesión les obliga a exponer sus creaciones o interpretaciones delante de un público en la mayoría de las ocasiones, se hace referencia a la ansiedad escénica musical.

En la actualidad, no existe una definición clara de ansiedad escénica musical lo que provoca que no tenga un único enunciado de referencia (Silva, 2007). No obstante, es un problema cuando el nivel de activación psicofisiológica de ansiedad supera el umbral soportado por el músico. Es entonces cuando sus recursos técnicos y su concentración disminuyen considerablemente, y tiene muy pocas probabilidades de volver a recuperar el nivel de interpretación alcanzado. Este hecho puede suceder de manera inesperada, aún a pesar no haber ocurrido experiencias previas (Osborne, Kenny, \& Holsomback, 2005). Además, afecta de manera clara a la actuación, encontrándose evidencias que correlacionan positivamente niveles altos de ansiedad con bajo logro en la interpretación musical (Yağişan, 2009).

Según Salmon (1990), la ansiedad escénica es vivida "como la experiencia de aprensión angustiosa sobre la disminución real de las habilidades interpretativas en un contexto público, hasta un grado que no garantice la aptitud musical del individuo, el entrenamiento o el nivel de preparación de éste" (p. 3); que puede resumirse en un evidente miedo a aparecer frente a una audiencia (American Psychiatric Association, 1994; Kenny, 2008; Studer, Danuser, Hildebrandt, Arial, \& Gomez, 2011 ) Para el intérprete, supone la sensación de niveles extremos de terror, cuya respuesta tiene tres componentes diferenciados: fisiológico (temblores de manos, taquicardia o sudoración excesiva), cognitivo (fallos de memoria, pensamientos negativos hacia la actuación y hacia sí mismos) y motor (evitación de actuaciones o abandono de carrera) (Lee, 2002; McAllister, 2011; Petrovich, 2004; Sandgren, 2002; Spahn, Echternach, Zander, Voltmer, \& Richter, 2010) y suelen precipitarse por la exposición pública y la percepción de 
una posible evaluación negativa por parte de los demás (Osborne \& McPherson, 2018 ; Taborsky, 2007). Según diversos estudios, los síntomas cognitivos, como la preocupación excesiva y problemas de concentración, son los que mayor malestar generan (Herrera, Jorge, \& Lorenzo, 2015; Kenny, Davis, \& Oates, 2004; Langendorfer, Hodapp, Kreutz, \& Bongard, 2006; Yağışhan, 2009).

Desde hace varios años, han sido numerosos artistas los que han salido al paso reconociendo que padecían ansiedad escénica, un problema que tradicionalmente se intentaba enmascarar y mantener en secreto. Actores, cantantes, vocalistas, músicos y orquestistas; no existe ninguna rama a la que no afecte este problema. De este modo, la ansiedad escénica musical es uno de los problemas más significativos a los que deben hacer frente durante su formación y práctica musical tanto el alumnado del Título Superior de Música como los músicos. Para muchos intérpretes puede suponer no sólo un impedimento, sino motivo de abandono de prometedoras carreras dentro de la interpretación musical (Lee, 2002; Wesner, Noyes, \& Davis, 1990). De hecho, existen estudios que confirman que el $20 \%$ de los músicos abandona su carrera por este motivo (Dalia, 2004). Además, tiene impacto sobre otras variables como, por ejemplo, la autoestima, donde la ansiedad escénica musical conlleva a un descenso considerable en el alumnado (Ramos, 2013). Desde un enfoque psicométrico, Sadler y Miller (2010) han encontrado que ciertos rasgos de personalidad, como el perfeccionismo, el neuroticismo, la ansiedad-rasgo, el pensamiento catastrofista y la fobia social, están estrechamente relacionados con la ansiedad escénica musical.

Algunos trabajos indican que el $20 \%$ de los músicos abandona su carrera por este motivo (Dalia, 2004), porcentaje que aumenta si se hace mención a edades preuniversitarias, donde la cifra, según Herrera y Jorge (2013), puede llegar hasta un 60-80\%. Por ello, resulta evidente la necesidad de realizar investigaciones para comprender la relación entre la actuación musical y la ansiedad escénica, para poder diseñar intervenciones en este campo.

Por otra parte, diversas investigaciones han demostrado la existencia de patrones de ansiedad diferentes en hombres y mujeres, de tal manera que las mujeres suelen presentar un nivel mayor de trastornos de ansiedad (Altemus, Sarvaiya, \& Epperson, 2014 ; Kenny \& Ackermann, 2015; Orgilés, Méndez, Espada, Carballo, \& Piqueras, 2012; Sandgren, 2009). Este patrón se repite cuando se hace referencia a enfrentarse a una actuación musical: también puntuan significativamente más alto las mujeres (Kenny et al., 2004; Rae \& McCambridge, 2004; Ryan, 2004; Yağişan, 2009; Yondem, 2007).

Sin embargo, las diferencias entre géneros no son constantes en todos los grupos de edad. Así, dichas diferencias van aumentando con la edad y, mientras que en la niñez estas diferencias no son significativas (Boucher \& Ryan, 2011 ), en la adolescencia empiezan a aparecer correlaciones positivas entre género femenino y ansiedad escénica musical, donde además las chicas presentan mayores niveles de angustia y dificultad (Kenny \& Osborne, 2006; Osborne \& Kenny, 2005, 2008), aunque dicha diferencia no es detectada por todos los estudios (Dempsey, 2015; Herrera et al., 2015).

Concretamente, el objetivo principal de la presente investigación es analizar la ansiedad escénica en una muestra de profesorado del Grado Superior de Música que, debido a su profesión, se ve expuesto a un gran número de conciertos y situaciones musicales que no siempre es capaz de manejar adecuadamente debido a la ansiedad que se produce por la actuación. Dicho objetivo se concreta en dos objetivos específicos. En primer lugar, identificar si el profesorado ha experimentado alguna vez, a lo largo de su carrera profesional, ansiedad escénica musical ante las actuaciones musicales. En segundo, determinar si la ansiedad escénica musical difiere en función del género.

Este tipo de investigaciones puede suscitar gran interés dentro de la comunidad psicológica y educativa musical española, pues se trata de un área que no ha recibido 
especial atención hasta las últimas décadas en nuestro país. La ansiedad escénica musical sí que ha sido ampliamente estudiada e investigada, en cambio, fuera de nuestras fronteras (lusca \& Dafinoiv, 2012; Robson \& Kenny, 2017).

\section{MÉTODO}

\section{PARTICIPANTES}

Para seleccionar a los participantes se ha utilizado un muestreo no probabilístico de tipo causal o incidental, puesto que se ha elegido directa e intencionalmente a los participantes en este estudio (Delgado \& Prieto, 2007), por su accesibilidad y la voluntad de colaborar. La muestra ha estado compuesta por un total de 46 docentes de Conservatorio pertenecientes a centros de Andalucía (Málaga, Sevilla) y de la ciudad autónoma de Melilla (España), de los cuales 34 eran hombres (73.9\%) y 12 mujeres (26.1\%). Su edad oscilaba de los 27 a los 56 años $(M=39.83 ; D T=7.26)$.

Los docentes indicaron que su experiencia como intérpretes se situaba entre los 2 y los 45 años $(M=23.57$; $D T=8.52)$. Respecto a los años de experiencia como docentes, el mínimo fue de 6 y el máximo de 31 años $(M=15.67 ; D T=6.76)$. En lo relativo a la especialidad instrumental, 26 (56.5\%) de los instrumentos de los participantes pertenecían a la familia de viento (clarinete, oboe, saxofón, fagot, trompeta y flauta travesera), $13(28.3 \%)$ a la de cuerda (guitarra, violín, viola y violonchelo), 6 (13.0\%) a la de teclado (piano) y $1(2.2 \%)$ a la de canto.

\section{INSTRUMENTOS DE MEDIDA}

En primer lugar, se diseñó una hoja de registro con una serie de datos sociodemográficos necesarios para describir a los participantes: género, edad, estudios musicales, figura laboral, años de experiencia como intérprete y años de experiencia como docente. Además, se establecieron diferentes cuestiones relativas a la ansiedad escénica musical, esto es, si se ha experimentado en algún momento ansiedad escénica musical; en el caso de ser positiva la respuesta a la cuestión anterior, qué fue lo más desagradable y cómo afrontó la situación en ese momento; también se le ofrecía un listado de estrategias de afrontamiento de la ansiedad escénica musical para conocer si habían utilizado alguna de ellas.

En segundo lugar, se empleó el Kenny Music Performance Anxiety Inventory (2004) (K-MPAI), validado en español por Zarza (2014). El K-MPAI es un instrumento diseñado por Kenny et al. (2004) y permite evaluar, desde el modelo de ansiedad de Barlow (2000), la ansiedad escénica presentando los tres factores o vulnerabilidades que pueden ser los generadores de la ansiedad: vulnerabilidad biológica, vulnerabilidad psicológica general y vulnerabilidad psicológica específica. El objetivo principal de dicho cuestionario es analizar los niveles de ansiedad general y específica en relación a las variables anteriormente mencionadas.

Está compuesta por un total de 26 ítems medidos en una escala Likert de 7 puntos, que van desde -3 (totalmente en desacuerdo) a 3 (totalmente de acuerdo). Así, puntuaciones altas en el ítem son directamente proporcionales al factor de vulnerabilidad correspondiente y viceversa. Los ítems del K-MPAI han sido redactados en su versión original para coincidir y mostrar coherencia con la teoría de la ansiedad de Barlow (2000). A partir de esto, Kenny (2009) agrupa los diferentes ítems del siguiente modo: factor 1 , el contexto de las experiencias tempranas, ítems 5, 9, 19, 21 y 24; factor 2: vulnerabilidad psicológica generalizada, ítems $1,2,3,4,6,8,10,11$, $15,16,17,18$ y 23; factor 3: preocupación por la actuación, ítems 7, 12, 13, 14, 20, 22, 25 y 26. La fiabilidad del K-MPAl, mediante la prueba de consistencia interna alpha de Cronbach, en el estudio de Kenny et al. (2004) es de .944. Por su parte, Zarza, Orejudo, Casanova y Mazas (2016) realizaron un análisis factorial confirmatorio con población española, poniendo de manifiesto la confirmación del modelo teórico de partida y las adecuadas propiedades psicométricas del instrumento. Asimismo encontraron que la fiabilidad del K-MPAl era de $\alpha=.866$. 


\section{PROCEDIMIENTO}

\section{RECOGIDA DE INFORMACIÓN}

Para realizar la presente investigación, se han seguido dos etapas claramente diferenciadas. La primera de ellas consistió en la traducción al español del K-MPAl así como la introducción previa de una serie de cuestiones sociodemográficas y relativas a la ansiedad escénica musical. Una vez realizado, se diseñó la versión online.

La segunda parte, fue la administración propiamente dicha, que se llevó a cabo de manera individual a través de un enlace online y que se ofreció de manera voluntaria al profesorado de Conservatorio de las ciudades anteriormente nombradas. Para ello, se contó con la colaboración del equipo directivo de cada Conservatorio. Además, se remitieron correos electrónicos a todo el profesorado, explicando en qué consistía el estudio y solicitando su colaboración. El cuestionario contaba con unas instrucciones claras al respecto, dejando cierto grado de autonomía para realizarlo. El tiempo medio de aplicación en los ensayos fue de 10-15 minutos.

\section{ANÁLISIS DE DATOS}

Para el análisis de los datos obtenidos a través del cuestionario se utilizó el paquete estadístico SPSS 20.0. Para las variables dependientes se determinó si se cumplía la normalidad de los datos, mediante la prueba de Kolmogorov-Smirnov. En la mayoría de los casos los resultados fueron significativos, $p<$ .05, por lo que no se cumplía la distribución gaussiana de los datos. Además, se halló que no se cumplía la homogeneidad de varianzas u homocedasticidad, atendiendo a la variable independiente género. Para ello, se utilizó el estadístico de Levene, resultando igualmente significativo. Por todo ello, y dado el tamaño de la muestra $(n=46)$, se optó por emplear pruebas no paramétricas en el análisis estadístico de los datos. De este modo, las pruebas estadísticas empleadas fueron el análisis de frecuencias, mediante la prueba
$\mathrm{Chi}^{2}$, así como $U$ de Mann-Whitney. En este último caso, se calculó el tamaño del efecto con la prueba la $r$ de Rosenthal.

\section{RESULTADOS}

Al preguntar a los participantes si han experimentado en algún ocasión ansiedad escénica musical, se observa que el $80.4 \%$ indica que sí (ver Tabla 1). El análisis de frecuencias, mediante el estadístico $\chi^{2}$, mostró diferencias significativas entre quienes experimentaban y no experimentaban ansiedad escénica musical $\left(\chi^{2}=17.043 ; p\right.$ $<.001)$.

Cuando se les preguntó qué fue lo más desagradable en esa situación de ansiedad escénica musical, las respuestas facilitadas se integran en la Tabla 2, siendo la pérdida de control (34.8\%) y los síntomas físicos (28.3\%) los más señalados. Se hallaron diferencias significativas en la frecuencia de respuestas $\left(\chi^{2}=32.522 ; p<.001\right)$.

Tabla 1

¿Has experimentado en alguna ocasión ansiedad escénica musical?

\begin{tabular}{|c|c|c|}
\hline & Frecuencia & Porcentaje \\
\hline Sí & 37 & 80.4 \\
\hline No & 9 & 19.6 \\
\hline Total & 46 & 100.0 \\
\hline
\end{tabular}

Tabla 2

¿Qué fue lo más desagradable para ti?

\begin{tabular}{|c|c|c|}
\hline & Frecuencia & Porcentaje \\
\hline Nada & 8 & 17.4 \\
\hline Síntomas físicos & 13 & 28.3 \\
\hline Síntomas cognitivos & 3 & 6.5 \\
\hline Pérdida de control & 16 & 34.8 \\
\hline Secuelas posteriores & 1 & 2.2 \\
\hline No rendir al máximo & 4 & 8.7 \\
\hline Público & 1 & 2.2 \\
\hline Total & 46 & 100.0 \\
\hline
\end{tabular}


Por otra parte, también se les preguntó cómo afrontaron dicha situación de ansiedad escénica musical. Las respuestas se incluyen en la Tabla 3 , siendo significativas las diferencias en las frecuencias de las diferentes formas de afrontar la situación de ansiedad escénica musical $\left(\chi^{2}=\right.$ $50.261 ; p<.001)$. Al respecto, el $37.0 \%$ señala que mediante ejercicios de respiración, el $21.7 \%$ que no hizo nada y el $19.6 \%$ que terminando la obra.

Igualmente, se solicitó a los participantes que señalasen si habían empleado alguna de las estrategias de afrontamiento de la ansiedad escénica musical que se recogen en la Tabla 4. Las dos más empleadas fueron el uso de fármacos
(23.9\%) y los ejercicios de respiración (21.7\%). Además, se hallaron diferencias significativas entre los que empleaban y no empleaban cada una de las estrategias de afrontamiento especificadas.

Respecto al K-MPAl, en la Tabla 5 se pueden consultar los porcentajes de respuesta para cada uno de los ítems que lo integran. El profesorado de Conservatorio señala que está Totalmente en desacuerdo en mayor medida con los siguientes ítems: A menudo siento que no tengo nada que esperar con ilusión (60.9\%), A menudo siento que no valgo mucho como persona (56.5\%), A menudo siento que la vida no tiene mucho que ofrecerme (43.5\%) y Cuando era niño, a menudo me sentía triste (43.5\%).

Tabla 3

¿Cómo afrontaste esta situación en ese mismo instante?

\begin{tabular}{|c|c|c|}
\hline & Frecuencia & Porcentaje \\
\hline Nada & 10 & 21.7 \\
\hline Terminando la obra & 9 & 2.2 \\
\hline Ayuda profesional & 1 & 37.0 \\
\hline Ejercicios de respiración & 17 & 6.5 \\
\hline Ejercicios cognitivos & 3 & 2.2 \\
\hline Ejercicios de relajación & 1 & 2.2 \\
\hline Rezando & 1 & 2.2 \\
\hline Parando la obra & 1 & 6.5 \\
\hline Otros & 3 & 100.0 \\
\hline Total & 46 & \\
\hline
\end{tabular}

Tabla 4

Estrategias de afrontamiento de la ansiedad escénica musical empleadas

\begin{tabular}{|c|c|c|c|c|}
\hline & Frecuencia & Porcentaje & $\chi^{2}$ & $p$ \\
\hline Fármacos & 11 & 23.9 & 12.522 & $<.001$ \\
\hline Alcohol & 0 & .0 & & $<.001$ \\
\hline Infusiones relajantes & 5 & 10.9 & 28.174 & \\
\hline Drogas no legales & 0 & .0 & & $<.001$ \\
\hline Ayuda psicológica & 2 & 4.3 & 38.348 & $<.001$ \\
\hline Ejercicios de respiración & 10 & 21.7 & 14.696 & $<.001$ \\
\hline Ejercicios de relajación muscular & 6 & 13.0 & 42.087 & $<.001$ \\
\hline Cuidado de los alimentos ingeridos & 1 & 2.2 & 37.356 & $<.001$ \\
\hline Yoga & 2 & 4.3 & 25.130 & $<.001$ \\
\hline Otros & 6 & 13.0 & 22.261 & $<.001$ \\
\hline Ninguna & 7 & 15.2 & & $<$ \\
\hline
\end{tabular}


Tabla 5

Porcentaje de docentes que elige cada opción de respuesta, en cada ítem del K-MPAI ( $N=46)$

\begin{tabular}{|c|c|c|c|c|c|c|c|}
\hline K-MPAI & -3 & -2 & -1 & 0 & 1 & 2 & 3 \\
\hline 1. Alguna vez siento que me deprimo sin saber por qué & 32.6 & 15.2 & 4.3 & 13.0 & 19.6 & 2.2 & 13.0 \\
\hline 2.Encuentro fácil confiar en los demás & 6.5 & 8.7 & 15.2 & 19.6 & 17.4 & 28.3 & 4.3 \\
\hline 3.Raramente siento que controlo mi vida & 28.3 & 32.6 & 8.7 & 13.0 & 6.5 & 6.5 & 4.3 \\
\hline $\begin{array}{l}\text { 4.A menudo tengo dificultades para generar la energía necesaria para hacer } \\
\qquad \text { cosas }\end{array}$ & 21.7 & 32.6 & 6.5 & 15.2 & 10.9 & 6.5 & 6.5 \\
\hline 5.La preocupación excesiva es una característica de mi familia & 13.0 & 21.7 & 13.0 & 17.4 & 6.5 & 17.4 & 10.9 \\
\hline 6.A menudo siento que la vida no tiene mucho que ofrecerme & 3.5 & 28.3 & 13.0 & 6.5 & 2.2 & 2.2 & 4.3 \\
\hline $\begin{array}{l}\text { 7.Incluso si trabajo mucho en la preparación para la actuación musical, es } \\
\text { probable que cometa errores }\end{array}$ & 4.3 & 23.9 & 19.6 & 10.9 & 17.4 & 17.4 & 6.5 \\
\hline 8.Encuentro difícil depender de otros & 8.7 & 6.5 & 8.7 & 21.7 & 15.2 & 26.1 & 13.0 \\
\hline 9. Mis padres fueron, la mayoría de las veces, receptivos a mis necesidades & 4.3 & .0 & 10.9 & 15.2 & 13.0 & 39.1 & 17.4 \\
\hline 10.Nunca sé antes de un concierto si mi actuación será buena & 15.2 & 23.9 & 15.2 & 8.7 & 7.4 & 10.9 & 8.7 \\
\hline 11.A menudo siento que no valgo mucho como persona & 56.5 & 21.7 & 6.5 & 4.3 & 4.3 & 4.3 & 2.2 \\
\hline $\begin{array}{l}\text { 12.Durante la actuación musical, me encuentro a mí mismo pensando si } \\
\qquad \text { conseguiré acabarla }\end{array}$ & 32.6 & 6.1 & 10.9 & 8.7 & 4.3 & 8.7 & 8.7 \\
\hline 13.Pensar en la evaluación puede tener interferencias en mi actuación & 15.2 & 13.0 & 6.5 & 8.7 & 23.9 & 17.4 & 15.2 \\
\hline $\begin{array}{l}\text { 14.Incluso en las situaciones en las que la actuación es más estresante, estoy } \\
\text { seguro de que actuaré bien }\end{array}$ & 6.5 & 8.7 & 8.7 & 32.6 & 21.7 & 15.2 & 6.5 \\
\hline $\begin{array}{l}\text { 15.A menudo me siento preocupado por una reacción negativa de la } \\
\text { audiencia }\end{array}$ & 17.4 & 17.4 & 17.4 & 13.0 & 17.4 & 10.9 & 6.5 \\
\hline 16.A veces me siento ansioso sin razón alguna & 19.6 & 21.7 & 10.9 & 13.0 & 19.6 & 6.5 & 8.7 \\
\hline $\begin{array}{l}\text { 17.Desde el inicio de mis estudios musicales, recuerdo estar ansioso por actuar } \\
\qquad \text { en público }\end{array}$ & 10.9 & 21.7 & 13.0 & 15.2 & 17.4 & 8.7 & 13.0 \\
\hline 18. Me preocupa que una mala actuación pueda arruinar mi carrera & 23.9 & 21.7 & 28.3 & 10.9 & 4.3 & 4.3 & 6.5 \\
\hline 19.Mis padres siempre me escuchaban & 6.5 & 10.9 & 2.2 & 15.2 & 28.3 & 10.9 & 26.1 \\
\hline $\begin{array}{l}\text { 20.Dejo pasar oportunidades de actuar que merecen la pena debido a la } \\
\qquad \text { ansiedad }\end{array}$ & 32.6 & 19.6 & 8.7 & 10.9 & 19.6 & 4.3 & 4.3 \\
\hline 21.Cuando era niño, a menudo me sentía triste & 43.5 & 30.4 & 15.2 & 4.3 & 2.2 & 2.2 & 2.2 \\
\hline $\begin{array}{l}\text { 22.A menudo me preparo para un concierto con la sensación de terror y } \\
\text { desastre inminente }\end{array}$ & 45.7 & 19.6 & 6.5 & 13.0 & 10.9 & .0 & 4.3 \\
\hline 23.A menudo siento que no tengo nada que esperar con ilusión & 60.9 & 19.6 & 4.3 & .0 & 8.7 & 2.2 & 4.3 \\
\hline 24.Mis padres me animaban a intentar cosas nuevas & 15.2 & 2.2 & 15.2 & 23.9 & 4.3 & 19.6 & 19.6 \\
\hline 25.Me preocupo tanto antes de una actuación musical, que no puedo dormir & 21.7 & 30.4 & 13.0 & 6.5 & 13.0 & 8.7 & 6.5 \\
\hline 26.Mi memoria suele ser muy fiable & 4.3 & 6.5 & 15.2 & 23.9 & 19.6 & 15.2 & 15.2 \\
\hline
\end{tabular}

Nota: -3 = Totalmente en desacuerdo; -2 = Bastante en desacuerdo; -1 = Un poco en desacuerdo; 0 = Ni de acuerdo ni en desacuerdo; $1=$ Un poco de acuerdo; 2 = Bastante de acuerdo; 3 = Totalmente de acuerdo 
Las respuestas al K-MPAl fueron analizadas por género. Los resultados se presentan en la Tabla 6. Al respecto, las mujeres puntuaron más alto que los hombres en los siguientes ítems: Alguna vez siento que me deprimo sin saber por qué $(U=117.00, p=.026, r=$ .329); Raramente siento que controlo mi vida $(U=114.50, p=.021, r=.340) ; A$ menudo tengo dificultades para generar la energía (U $=115.50, p=.023, r=.335) ; A$ menudo siento que no valgo mucho como persona $(U$ $=113.00, p=.011, r=.373)$; y Durante la actuación musical, me encuentro a mí mismo pensando si conseguiré acabarla $(U=$ $112.00, p=.018, r=.349$ ).

Tabla 6

Resultados de la prueba $U$ de Mann-Whitney en el K-MPAl según el género $(N=46)$

\begin{tabular}{|c|c|c|c|c|c|c|c|}
\hline K-MPAI & Género & $n$ & $\begin{array}{c}\text { Rango } \\
\text { promedio }\end{array}$ & $\begin{array}{l}\text { Suma de } \\
\text { rangos }\end{array}$ & $U$ & $p$ & $r$ \\
\hline \multirow{2}{*}{$\begin{array}{l}\text { Alguna vez siento que me } \\
\text { deprimo sin saber por qué }\end{array}$} & Hombre & 34 & 20.94 & 712.00 & $117.00 *$ & .026 & .329 \\
\hline & Mujer & 12 & 30.75 & 369.00 & & & \\
\hline \multirow{2}{*}{$\begin{array}{l}\text { Encuentro fácil confiar en los } \\
\text { demás }\end{array}$} & Hombre & 34 & 22.76 & 774.00 & 179.00 & .523 & .094 \\
\hline & Mujer & 12 & 25.58 & 307.00 & & & \\
\hline \multirow{2}{*}{$\begin{array}{l}\text { Raramente siento que controlo } \\
\text { mi vida }\end{array}$} & Hombre & 34 & 20.87 & 709.50 & $114.50 *$ & .021 & .340 \\
\hline & Mujer & 12 & 30.96 & 371.50 & & & \\
\hline \multirow{2}{*}{$\begin{array}{c}\text { A menudo tengo dificultades para } \\
\text { generar la energía necesaria para } \\
\text { hacer cosas }\end{array}$} & Hombre & 34 & 20.90 & 710.50 & $115.50 *$ & .023 & .335 \\
\hline & Mujer & 12 & 30.88 & 370.50 & & & \\
\hline \multirow{2}{*}{$\begin{array}{l}\text { La preocupación excesiva es una } \\
\text { característica de mi familia }\end{array}$} & Hombre & 34 & 23.49 & 798.50 & 203.50 & .990 & .001 \\
\hline & Mujer & 12 & 23.54 & 282.50 & & & \\
\hline \multirow{2}{*}{$\begin{array}{l}\text { A menudo siento que la vida no } \\
\text { tiene mucho que ofrecerme }\end{array}$} & Hombre & 34 & 22.22 & 755.50 & 160.50 & .250 & .169 \\
\hline & Mujer & 12 & 27.13 & 325.50 & & & \\
\hline \multirow{2}{*}{$\begin{array}{c}\text { Incluso si trabajo mucho en la } \\
\text { preparación para la actuación } \\
\text { musical, es pro probable que } \\
\text { cometa errores }\end{array}$} & Hombre & 34 & 22.59 & 768.00 & 173.00 & .430 & .116 \\
\hline & Mujer & 12 & 26.08 & 313.00 & & & \\
\hline
\end{tabular}




\begin{tabular}{|c|c|c|c|c|c|c|c|}
\hline Resultados de la & veba Ud & $\begin{array}{l}\text { bla } \\
\text { inn- }\end{array}$ & $\begin{array}{l}\text { Continuaciór } \\
\text { ney en el K. }\end{array}$ & PAl según e & énero (N & & \\
\hline K-MPAI & Género & $n$ & $\begin{array}{c}\text { Rango } \\
\text { promedio }\end{array}$ & $\begin{array}{l}\text { Suma de } \\
\text { rangos }\end{array}$ & $U$ & $p$ & $r$ \\
\hline & Hombre & 34 & 23.74 & 807.00 & 196.00 & .839 & .030 \\
\hline Otros & Mujer & 12 & 22.83 & 274.00 & & & \\
\hline Mis padres fueron, la mayoría & Hombre & 34 & 23.51 & 799.50 & 203.50 & .990 & .001 \\
\hline necesidades & Mujer & 12 & 23.46 & 281.50 & & & \\
\hline & Hombre & 34 & 22.00 & 748.00 & 153.00 & .196 & .190 \\
\hline mi actuación será buena & Mujer & 12 & 27.75 & 333.00 & & & \\
\hline A menudo siento que no valgo & Hombre & 34 & 20.82 & 708.00 & $113.00^{*}$ & .011 & .373 \\
\hline mucho como persona & Mujer & 12 & 31.08 & 373.00 & & & \\
\hline Durante la actuación musical, me & Hombre & 34 & 20.79 & 707.00 & $112.00 *$ & .018 & .349 \\
\hline si conseguiré acabarla & Mujer & 12 & 31.17 & 374.00 & & & \\
\hline 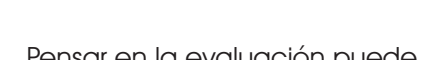 & Hombre & 34 & 21.56 & 733.00 & 138.00 & .094 & .247 \\
\hline actuación & Mujer & 12 & 29.00 & 348.00 & & & \\
\hline Incluso en las situaciones en & Hombre & 34 & 24.82 & 844.00 & 159.00 & .248 & .170 \\
\hline actuaré bien & Mujer & 12 & 19.75 & 237.00 & & & \\
\hline & Hombre & 34 & 23.90 & 812.50 & 190.50 & .732 & .050 \\
\hline audiencia & Mujer & 12 & 22.38 & 268.50 & & & \\
\hline & Hombre & 34 & 22.71 & 772.00 & 177.00 & .493 & .101 \\
\hline & Mujer & 12 & 25.75 & 309.00 & & & \\
\hline
\end{tabular}


Tabla 6 (Continuación)

Resultados de la prueba $U$ de Mann-Whitney en el K-MPAl según el género $(N=46)$

\begin{tabular}{|c|c|c|c|c|c|c|c|}
\hline K-MPAI & Género & $n$ & $\begin{array}{l}\text { Rango } \\
\text { promedio }\end{array}$ & $\begin{array}{l}\text { Suma de } \\
\text { rangos }\end{array}$ & $U$ & $p$ & $r$ \\
\hline \multirow{2}{*}{$\begin{array}{l}\text { Desde el inicio de mis estudios } \\
\text { musicales, recuerdo estar ansioso } \\
\text { por actuar en público }\end{array}$} & Hombre & 34 & 23.22 & 789.50 & 194.50 & .810 & .035 \\
\hline & Mujer & 12 & 24.29 & 291.50 & & & \\
\hline \multirow{2}{*}{$\begin{array}{l}\text { Me preocupa que una mala } \\
\text { actuación pueda arruinar mi } \\
\text { carrera }\end{array}$} & Hombre & 34 & 23.10 & 785.50 & 190.50 & .729 & .051 \\
\hline & Mujer & 12 & 24.63 & 295.50 & & & \\
\hline \multirow{2}{*}{$\begin{array}{l}\text { Mis padres siempre me } \\
\text { escuchaban }\end{array}$} & Hombre & 34 & 23.12 & 786.00 & 191.00 & .739 & .049 \\
\hline & Mujer & 12 & 24.58 & 295.00 & & & \\
\hline \multirow{2}{*}{$\begin{array}{l}\text { Dejo pasar oportunidades de } \\
\text { actuar que merecen la pena } \\
\text { debido a la ansiedad }\end{array}$} & Hombre & 34 & 22.96 & 780.50 & 185.50 & .635 & .070 \\
\hline & Mujer & 12 & 25.04 & 300.50 & & & \\
\hline \multirow{2}{*}{$\begin{array}{l}\text { Cuando era niño, a menudo me } \\
\text { sentía triste }\end{array}$} & Hombre & 34 & 24.03 & 817.00 & 186.00 & .632 & .070 \\
\hline & Mujer & 12 & 22.00 & 264.00 & & & \\
\hline \multirow{2}{*}{$\begin{array}{l}\text { A menudo me preparo para un } \\
\text { concierto con la sensación de } \\
\text { terror y desastre inminente }\end{array}$} & Hombre & 34 & 22.10 & 751.50 & 156.50 & .209 & .185 \\
\hline & Mujer & 12 & 27.46 & 329.50 & & & \\
\hline \multirow{2}{*}{$\begin{array}{l}\text { A menudo siento que no tengo } \\
\text { nada que esperar con ilusión }\end{array}$} & Hombre & 34 & 22.22 & 755.50 & 160.50 & .214 & .183 \\
\hline & Mujer & 12 & 27.13 & 325.50 & & & \\
\hline \multirow{2}{*}{$\begin{array}{l}\text { Mis padres me animaban a } \\
\text { intentar cosas nuevas }\end{array}$} & Hombre & 34 & 22.26 & 757.00 & 162.00 & .285 & .157 \\
\hline & Mujer & 12 & 27.00 & 324.00 & & & \\
\hline \multirow{2}{*}{$\begin{array}{c}\text { Me preocupo tanto antes de una } \\
\text { actuación musical, que no puedo } \\
\text { dormir }\end{array}$} & Hombre & 34 & 22.34 & 759.50 & 164.50 & .312 & .149 \\
\hline & Mujer & 12 & 26.79 & 321.50 & & & \\
\hline \multirow{2}{*}{ Mi memoria suele ser muy fiable } & Hombre & 34 & 24.19 & 822.50 & 180.50 & .550 & .088 \\
\hline & Mujer & 12 & 21.54 & 258.50 & & & \\
\hline
\end{tabular}




\section{DISCUSIÓN}

El presente trabajo ha tenido como uno de sus objetivos identificar si el profesorado ha experimentado alguna vez, a lo largo de su carrera profesional, ansiedad escénica musical ante las actuaciones musicales. Tal y como se desprende de los resultados hallados, se cumple la hipótesis de partida de que los docentes de Conservatorio experimentan ansiedad escénica musical. Asimismo, otro objetivo era determinar si la ansiedad escénica musical difiere en función de la variable género, poniéndose de manifiesto que las mujeres puntúan más alto que los hombres en determinados ítems relacionados con la ansiedad escénica musical. Seguidamente se discutirán en mayor profundidad cada uno de estos aspectos.

Los resultados generales de la investigación coinciden con los de Silva (2007), para quien este problema sigue sin tener aún hoy en día una definición plenamente asentada. Sin embargo, sí existe consenso entre la comunidad científica en que, definiciones aparte, está formada por componentes fisiológicos, cognitivos, motores, sociales, conductuales, además de que es un problema presente en la población de músicos española y mundial, tal y como se ha comprobado en el presente estudio, donde un porcentaje superior al $80 \%$ del profesorado de conservatorio ha afirmado padecer en alguna ocasión ansiedad escénica musical. Este resultado corrobora los hallados en otros estudios (Dalia, 2004; Herrera \& Jorge, 2013; Zarza et al., 2016).

Tal y como apuntan numerosos estudios, los participantes coinciden en señalar los síntomas cognitivos, como la pérdida de control, como los síntomas más desagradables experimentados durante la experiencia de ansiedad escénica musical (Herrera et al., 2015, Kenny et al., 2004; Kenny \& Halls, 2018; Langendorfer et al., 2006; Yağışhan, 2009). Con un porcentaje considerable, les siguen los síntomas físicos, siendo en la mayoría de ellos, los temblores.

También, al comprobar la manera de afrontar dicha situación de ansiedad escénica musical, los resultados apuntan a que, la mayor parte, realiza ejercicios de respiración, seguidos de los que no utilizaban ninguna estrategia o de los que decidieron terminar la obra con resignación. Estos datos concuerdan con los obtenidos por Biasutti y Concina (2014) ya que la mayoría de los participantes adoptó estrategias para enfrentar el problema.

Un resultado significativo fue el obtenido respecto a las estrategias empleadas frente a la ansiedad escénica musical, donde la principal fue la ingesta de fármacos, seguida de los ejercicios de respiración. Ya Fishbein, Middlestadt, Ottati, Straus y Ellis (1988) documentaron la toma de medicamentos como una estrategia frecuente usada para reducir la ansiedad escénica musical, que posteriormente ha sido corroborada por numerosos autores (Park, 2010; Tamborrino, 2001 ; Zarza, 2014). Aún así, las técnicas de relajación siguen siendo las más utilizadas según las fuentes consultadas (Biasutti \& Gilda, 2006; Fishbein et al., 1988).

En lo que respecta al segundo objetivo de investigación, las mujeres obtienen mayores puntuaciones en los factores preocupación por la actuación y vulnerabilidad psicológica generalizada; lo que coincide con las propuestas por Barlow (2000). Esto indicaría una mayor predisposición del género femenino a padecer trastornos de ansiedad, en general, y ansiedad escénica, en particular, debido a que padecen una mayor indefensión y presencia de pensamientos negativos. Estos resultados concuerdan con la mayoría de la literatura revisada en el presente estudio (Altemus et al., 2014; Kenny \& Ackermann, 2015; Orgilés et al., 2012 ; Sandgren, 2009).

En definitiva, la presente investigación ha permitido describir la ansiedad escénica musical en una muestra de profesorado de Conservatorio ya que, como apunta Demirbartir (2012), este problema está muy presente dentro de la comunidad musical, es el principal motivo por el que las interpretaciones de los músicos se ven deterioradas y puede incluso arruinar prometedoras carreras (David, 2010). Como ya ha sido descrito, la ansiedad escénica musical amenaza a una parte considerable de la población de músicos profesionales y 
profesorado de música alrededor del mundo. El estudio de la misma desde las etapas de formación superior o universitaria es clave para prevenirla e introducir mejoras en los ámbitos normativo y pedagógico. No se debe olvidar que hay estudios que califican la profesión de músico como una de las más estresantes y que guarda mayor relación con problemas afectivos (Orejudo, Nuño, Fernández, Ramos, \& Herrero, 2006; van Fenema, Julsing, \& Carlier, 2012). El motivo principal es el gran número de estresores a los que se ven expuestos, los cuales han sido descritos anteriormente, a los que habría que sumar el carácter competitivo típico de las enseñanzas musicales, los viajes frecuentes a sitios desconocidos y en solitario, la necesidad de obtener premios e invitaciones a festivales. Todo ello puede llegar a convertirse en un sentimiento de incontrolabilidad, tal y como describió Barlow (2000) en su teoría de los tres factores de la ansiedad.

Diversos autores han dado cuenta de la importancia de ofrecer una enseñanza específica en ansiedad escénica en las etapas de formación y que ésta se imparta de una manera reglada, formando parte del currículo (Bujez, 2008; Cid, 2012; Yağişhan, 2009). El principal motivo, además de mejorar la práctica musical, es que una gran parte de estos músicos en formación pasarán a ser docentes en un futuro y se ha hecho patente la necesidad de dotar de herramientas para el mejor desempeño de la práctica pedagógica del instrumento musical. En la actualidad, la educación musical española se caracteriza por una formación específica escasa en lo que respecta al hecho de afrontar las exposiciones públicas, tan solo compuesta por algunos consejos que carecen del suficiente rigor y contraste, al margen de cualquier plan docente previamente establecido. Por lo tanto, se ha de atender a una mejora de la formación musical española, proponiendo otras líneas pedagógicas, curriculares y psicológicas para obtener mejores profesionales de la música.

Referente a lo anterior, las investigaciones musicales también están sufriendo cambios sustanciales, sobre todo en el entorno occidental (Hargreaves \& Marshall, 2005), incorporando un enfoque interdisciplinar y a limentándose de diferentes teorías para actuar en el hecho musical en su conjunto. Ámbitos como la Psicología Cognitiva, la Psicología Social, la Filosofía o la Etnomusicología son algunos de los nuevas perspectivas propuestas por diferentes autores (Hargreaves \& Marshall, 2005; Ibarretxe, 2006). De hecho, la Psicología tiene una vital importancia en las artes, especialmente en la educación musical y en las destrezas musicales ya que condiciona diferentes componentes como son el cognitivo, afectivo y psicomotriz (Hargreaves \& North, 1997). Como consecuencia, las diferentes teorías y técnicas psicológicas van a desempeñar un papel importante en la formulación y evaluación de métodos de la enseñanza musical. Durante todo su periodo de formación tanto el alumnado de grado superior como el profesorado debe hacer frente a numerosas situaciones en público ya que ser músico lleva inevitablemente a al hecho de ofrecer conciertos y audiciones $y$, tanto en su formación como en su profesión, a ser evaluados por terceros.

Para finalizar, en lo que respecta al estudio presentado, se deben contemplar una serie de limitaciones que hacen necesaria la replicación de investigaciones que ratifiquen o refuten los resultados presentados, además de que sean útiles para incrementar el conocimiento que se tiene de la ansiedad escénica musical en profesorado de Conservatorio. En la investigación desarrollada una de sus limitaciones radica en la muestra, ya que no se ha podido realizar una aleatorización de la población y no es demasiado numerosa, en términos absolutos y comparados con el total de profesorado que imparte clase en los conservatorios de nuestro país. Sin embargo, hay que tener en cuenta el hecho de que se haya elegido al profesorado en lugar de al alumnado de grado superior, ya que la literatura específica acerca de este colectivo es menor, por lo que es un campo que merece la misma atención que la del alumnado en formación. Este hecho, lejos de invalidar los resultados, puede servir para plantear nuevas investigaciones que mejoren esta limitación. 
En lo que concierne a las variables estudiadas, sería necesario ampliar el número de variables para los estudios posteriores (edad, especialidad instrumental, años de experiencia como instrumentista o flow), de modo que se pueda profundizar en mayor medida en las relaciones existentes entre ellas, reforzando así los resultados. Por último, respecto al instrumento de recogida de información utilizado, a pesar de estar bastante aceptado por la comunidad científica y permitir un análisis exhaustivo del constructo de la ansiedad escénica y su relación con diferentes constructos psicológicos, se podrían incluir en futuras investigaciones un mayor número de instrumentos, entre ellos, entrevistas, lo que permitiría avanzar hacia una metodología mixta de investigación (Bergman, 2010; Creswell \& Plano, 2007).

\section{- Conflicto de intereses.}

Los autores declaran no tener ningún conflicto de intereses.

\section{REFERENCIAS}

Altemus, M., Sarvaiya, N., \& Epperson, C. (2014). Sex differences in anxiety and depression clinical perspectives. Frontiers in Neuroendocrinology, 35(3), 320-330. doi:10.1016/i.yfrne.2014.05.004

American Psychiatric Association (1994). Diagnostic and Statistical Manual of Mental Disorders (4th ed.). Washington, DC: American Psychiatric Association.

Ballester, J. (2015). Un estudio de la ansiedad escénica en los músicos de los conservatorios de la Región de Murcia (Tesis Doctoral, Universidad de Murcia). Recuperado de https://digitum.um.es/ xmlui/handle/10201/45626

Barlow, D.H. (2000). Unraveling the mysteries of anxiety and its disorders from the perspective of emotion theory. American Psychologist, 55(1 1), 1247-1259.

Bergman, M.M. (2010). On concepts and paradigms in mixed methods research. Journal of Mixed Methods Research, 4(3), 171-175. doi: $10.1177 / 1558689810376950$

Biasutti, M., \& Concina, E. (2014). The role of coping strategy and experience in predicting music performance anxiety. Musicae Scientiae, 18(2), 189-202. doi:10.1177/1029864914523282

Boucher, H., \& Ryan, C.A. (2011). Performance stress and the very young musician. Journal of Research in Music Education, 58(4), 329. 345.

Bújez, A.V. (2008). La LOGSE en los Conservatorios Superiores de Música de Andalucía: Una reflexión sobre el currículo. Sevilla: Centro de Documentación Musical de Andalucía.

Cid, M.J. (2012) Los conservatorios superiores de Galicia durante la LOGSE. Revista Electrónica de LEEME (lista europea electrónica de música en la educación), 28, 1-22. Recuperado de http://musica.rediris.es/leeme/revista/cid12. pdf

Creswell, J.W., \& Plano, V.L. (2007). Designing and conducting Mixed Methods research. Thousand Oaks, CA: Sage.

Dalia, G. (2004). Cómo superar la ansiedad escénica en músicos. Madrid: Mundimúsica Ediciones.

David, A.P. (2010). Examining the relationship of personality and burnout in college students: The role of academic motivation. Educational Measurement and Evaluation Review, 1, 90104.

Delgado, A.R., \& Prieto, G. (2007). Introducción a los Métodos de Investigación en Psicología. Madrid: Pirámide.

Demirbatir, R.E. (2012). Undergraduate music students' depression, anxiety and stress levels: a study from Turkey. Procedia. Social and Behavioral Sciences, 46, 2995-2999. doi:10.1016/i.sbspro.2012.05.603

Dempsey, E. (2015). Music Performance Anxiety in Children and Teenagers: Effects of Perfectionism, Self-Efficacy, and Gender (Doctoral Thesis, University of Ottawa).

Fishbein, M., Middlestadt, S.E., Ottati, V., Straus, S., \& Ellis, A. (1988). Medical problems among ICSOM musicians: Overview of a national survey. Medical Problems of Performing Artists, 3, 1-8.

Hallam, S., Cross, I., \& Thaut, M.H. (2011). Oxford Handbook of Music Psychology. Oxford, UK: Oxford University Press.

Hargreaves, D.J., \& Marshall, N.A. (2005). 
Educación musical en el siglo XXl: una perspectiva psicológica. Eufonía. Didáctica de la Música, 34, 8-33.

Hargreaves, D.J., \& North, A.C. (1997). The social psychology of music. Oxford, UK: Oxford University Press.

Herrera, L., \& Jorge, G. (2013). Ansiedad escénica musical en estudiantes de flauta travesera. Eufonía. Didáctica de la música. 57, 43-55.

Herrera, L., Jorge, G., \& Lorenzo, O. (2015). Ansiedad escénica musical en alumnos de flauta travesera de conservatorio. Revista Mexicana de Psicología, 32(2), 169-181.

Ibarretxe, G. (2006). El conocimiento científico en investigación musical. En M. Díaz (Coord.), Introducción a la investigación en Educación Musical (pp. 8-30). Madrid: Enclave Creativa.

lusca, D., \& Dafinoiv, I. (2012). Performance anxiety and musical level of undergraduate students in exam situations: the role of gender and musical instrument. Procedia. Social and Behavioral Sciences, 33, 448-452. doi:10.1016/i.sbspro.2012.01.161

Kenny, D.T. (2008). Music Performance Anxiety. International Handbook of Musicians' Health and Wellbeing. Oxford, UK: Oxford University Press.

Kenny, D.T. (2009). The factor structure of the revised Kenny Music Performance Anxiety Inventory. International Symposium on Performance Science, 37-41. Retrieved from http://www.performancescience.org/cache/ fl0019647.pdf

Kenny, D.T., \& Ackermann, B. (2015). Performance-related musculoskeletal pain, depression and music performance anxiety in professional orchestral musicians. A population study. Psychology of Music, 43(1), 43-60. doi:10.1177/0305735613493953

Kenny, D.T., Davis, P., \& Oates, J. (2004). Music performance anxiety and occupational stress amongst opera chorus artists and their relationship with state and trait anxiety and perfectionism. Journal of Anxiety Disorders, 18(6), 757-777. doi:10.1016/i. janxdis.2003.09.004

Kenny, D.T., \& Halls, N. (2018). Development and evaluation of two brief group interventions for music performance anxiety in community musicians. Psychology of Music, 46(1), 6683. doi:10.1177/0305735617702536

Kenny, D.T., \& Osborne, M.S. (2006). Music performance anxiety: New insights from young musicians. Advances in Cognitive Psychology, 2(2), 103-112. doi:10.2478/v10053-0080049-5

Langendorfer, F., Hodapp, V., Kreutz,G.,\&Bongard, S. (2006). Personality and performance anxiety among professional orchestra musicians. Journal of Individual Differences, 27(3), 162171. doi:10.1027/1614-0001.27.3.162.

Lee, S. (2002). Musician's performance anxiety and coping strategies. American Music Teacher, 52(1), 36-39.

McAllister, J.A. (2011). Performance Anxiety Amongst Middle School- Aged Wind Instrumentalists as Influenced by Variations in Delivery of Instructional Script Given by Adjudicators During Sight Reading (Master Thesis, Florida International University).

Orejudo, S., Nuño, J., Fernández, T., Ramos, M.T., \& Herrero, M.L. (2006). Miedo a hablar en público. Implicaciones para el entorno académico en un contexto de reforma universitaria. Revista de Psicología General y Aplicada, 59, 257-276.

Orgilés, M., Méndez, X., Espada, J.P., Carballo, J.L., \& Piqueras, J.A. (2012). Anxiety disorder symptoms in children and adolescents: Differences by age and gender in a community sample. Revista de Psiquiatría y Salud Mental (English Edition), 5(2), 1 15-120. doi:10.1016/i.rpsm.2012.01.005

Osborne, M.S., \& Kenny, D.T. (2005). Development and validation of a music performance anxiety inventory for giffed adolescent musicians. Journal of Anxiety Disorders, 19(7), 725-751. doi:10.1016/i. janxdis.2004.09.002

Osborne, M.S., \& Kenny, D.T. (2008). The Role of Sensitizing Experiences in Music Performance Anxiety in Adolescent Musicians. Psychology of Music, 36(4), 447-462. doi:10.1177/0305735607086051

Osborne, M.S., Kenny, D.T., \& Holsomback, R. (2005). Assessment of MPA in late childhood: A validation study of the Music Performance Anxiety Inventory for Adolescents (MPAI-A). International Journal of Stress Management, 
12, 312-330. doi:10.1037/10725245.12.4.312

Osborne, M.S., \& McPherson, G.E. (2018). Precompetitive appraisal, performance anxiety and confidence in conservatorium musicians: A case for coping. Psychology of Music. Advance online publication. doi:10.1177/0305735618755000

Papageorgi, I., Creech, A., \& Welch, G. (2011). Perceived performance anxiety in advanced musicians specializing in different musical genres. Psychology of Music, 47(1), 18-41. doi:10.1177/0305735611408995

Papageorgi, I., Hallam, S., \& Welch, G.F. (2007). A conceptual framework for understanding musical performance anxiety. Research Studies in Music Education, 28(1), 83-107. doi:10.11 77/1321103X070280010207

Park, J.E. (2010). The Relationship between Musical Performance Anxiety, Healthy Lifestyle Factors, and Substance Use among Young Adult Classical Musicians: Implications for Training and Education. Columbia: Columbia University.

Petrovich, A. (2004). A. Performance anxiety: how teachers can help. American Music Teacher, 53(3), 24-27.

Rae, G., \& McCambridge, K. (2004). Correlates of Performance Anxiety in Practical Music Exams. Psychology of Music, 32(4), 432-439. doi: $10.1177 / 0305735604046100$

Ramos, P. (2013). La Validez y la Eficacia de los Ejercicios Respiratorios para Reducir la Ansiedad Escénica en el Aula de Música. Revista Internacional de Educación Musical, l, 23-30. doi:10.12967/RIEM-20131-p023-030

Robson, K.E., \& Kenny, D.T. (2017). Music performance anxiety in ensemble rehearsals and concerts: A comparison of music and non-music major undergraduate musicians. Psychology of Music, 45(6), 868-885. doi:10.1177/0305735617693472

Ryan, C. (2004). Gender Differences in Children's Experience of Musical Performance Anxiety. Psychology of Music, 32(1), 89-103. doi:10.1017/CBO9781 107415324.004

Sadler, M.E., \& Miller, C.J. (2010). Performance Anxiety: A Longitudinal Study of the Roles of Personality and Experience in Musicians. Social Psychological and Personality Science, 1(3), 280-287. doi:10.1177/1948550610370492

Salmon, P. (1990). A psychological perspective on Musical Performance Anxiety - a review of the literature. Medical Problems of Performing Artists, 5(1), 1-11.

Sandgren, M. (2002). Voice, Soma, and Psyche: A Qualitative and Quantitative Study of Opera Singers. Medical Problems of Performing Artists, 17, 11-21.

Sandgren, M. (2009). Health Anxiety Instead of Performance Anxiety among Opera Singers. Proceedings of the 7th Triennial Conference of European Society for the Cognitive Sciences of Music, 468-474.

Silva, L, (2007). Miedo escénico en músicos académicos de Caracas: Una aproximación psicosocial desde el análisis del discurso (Trabajo Fin de Grado, Universidad Central de Venezuela). Recuperado de http://www. musicaenclave.com/trabajosdegradopdf/ tesislaurasilva.pdf

Spahn, C., Echternach, M., Zander, M.F., Voltmer, E., \& Richter, B. (2010). Music performance anxiety in opera singers. Logopedics Phoniatrics Vocology, 35(4), 175 182. doi: $10.3109 / 14015431003720600$

Studer, R., Danuser, B., Hildebrandt, H., Arial, M., \& Gomez, P. (2011). Hyperventilation complaints in music performance anxiety among classical music students. Journal of Psychosomatic Research, 70(6), 557-564. doi:10.1016/i.jpsychores.2010.11.004

Taborsky, C. (2007). Musical performance anxiety: A review of literature. Update: Applications of Research in Music Education, 26(1), 15-25. doi:10.1177/875512330702 60010103

Tamborrino, R. A. (2001). An examination of performance anxiety associated solo performance of college level music majors. Dissertation Abstracts International Section A: Humanities and Social Sciences, 62, (5-A).

Van Fenema, E., Julsing, J., \& Carlier, I. (2012). Musicians Seeking Psychiatric Help; A Closer Look At Performance Anxiety. European Psychiatry, 27(1), 1. doi:10.1016/S09249338(12)74323-7.

Wesner, R.B., Noyes, R.Jr., \& Davis, T.L. (1990). 
The occurrence of performance anxiety among musicians. Journal of Affective Disorders, 18(3), 177-185. doi:10.1016/01650327(90)90034-6

Yağişan, N. (2009). A Survey of Music Performance Anxiety. Turkish Journal Music Education, 2(1), 1-11.

Yondem, Z.D. (2007). Performance Anxiety, Dysfunctional Attitudes and Gender in University Music Students. Social Behavior and Personality, 35(10), 1415-1426. doi:10.2224/sbp.2007.35.10.1415

Zarza, F.J. (2014). Variables psicológicas y pedagógicas como predictoras de la ansiedad escénica en estudiantes de grado superior de música de España (Tesis Doctoral, Universidad de Zaragoza). Recuperado de https://zaguan. unizar.es/record/47413
Zarza, F.J., Casanova, O., \& Orejudo, S. (2016). Modelo explicativo de la ansiedad escénica en estudiantes de instrumentos de viento madera del Título Superior de Música. Sinfonía Virtual. Revista de Música y Reflexión Musical, 31, 1-13. Recuperado de http://www. sinfoniavirtual.com/revista/031/ansiedad.pdf Zarza, F.J., Casanova, O., \& Robles, J.E. (2016). Relación entre ansiedad escénica, perfeccionismo y calificaciones en estudiantes del Título Superior de Música. ReiDoCrea, 5, 16-21.

Zarza, F.J., Orejudo, S., Casanova, O., \& Mazas, B. (2016). Kenny Music Performance Anxiety Inventory: Confirmatory factor analysis of the Spanish version. Psychology of Music, 44(3), 340-352. doi:10.1177/0305735614567932 